\title{
THE JONES POLYNOMIAL OF PERIODIC KNOTS
}

\author{
YOSHIYUKI YOKOTA
}

(Communicated by Frederick R. Cohen)

\begin{abstract}
We give the conditions for the Jones polynomial of periodic knots which are the improvement of Traczyk's and Murasugi's results.
\end{abstract}

\section{INTRODUCTION}

A knot $K$ in $S^{3}$ is said to have period $r>1$, if there exists an orientation preserving homeomorphism $f$ on $S^{3}$ of period $r$ which preserves $K$ with $\operatorname{Fix}(f) \cong S^{1}$ and $\operatorname{Fix}(f) \cap K=\varnothing$. By the positive solution of Smith Conjecture, $\operatorname{Fix}(f)$ is unknotted. Let $\Sigma^{3}$ be the quotient space under $f$ and $\varphi: S^{3} \rightarrow \Sigma^{3}$ the quotient map. Then $\Sigma^{3}$ is a 3 -sphere. We call $\varphi(K)$, denoted by $k$, the factor knot of $K$.

Recently, some results concerning the Jones polynomial have been applied to the study of periodic knots [6,7]. In this paper, we give more precise conditions of the Jones polynomial of periodic knots. In fact, we will prove the following theorems. Here we denote the Jones polynomial of a knot $K$ by $V_{K}(t)$.

Theorem 1. For an odd prime $r$, let $K$ be an $r$ periodic knot and $f$ the periodic map on $S^{3}$ realizing the period.

(i) If $\operatorname{lk}(K, \operatorname{Fix}(f)) \equiv 1 \bmod 2$, then

$$
V_{K}(t)-V_{K}\left(t^{-1}\right) \equiv 0 \bmod \left(r, t^{2 r}-1\right) .
$$

(ii) If $\operatorname{lk}(K, \operatorname{Fix}(f)) \equiv 0 \bmod 2$, then

$$
V_{K}(t)-V_{K}\left(t^{-1}\right) \equiv 0 \bmod \left(r, t^{r}-1\right)
$$

and

$$
V_{K}(t)+V_{K}\left(t^{-1}\right) \equiv 0 \bmod \left(r,\left(t^{r}+1\right) /(t+1)\right) .
$$

Received by the editors March 27, 1990.

1980 Mathematics Subject Classification (1985 Revision). Primary 57M25.

Key words and phrases. Jones polynomial, periodic knot. 
Theorem 2. Under the same assumption as in Theorem 1 , let $k$ be the factor knot of $K$.

(i) If $\operatorname{lk}(K, \operatorname{Fix}(f)) \equiv 1 \bmod 2$, then

$$
V_{K}(t) \equiv\left[V_{k}(t)\right]^{r} \bmod \left(r, t^{2 r}-t^{r+1}-t^{r-1}+1\right) .
$$

(ii) If $\operatorname{lk}(K, \operatorname{Fix}(f)) \equiv 0 \bmod 2$, then

$$
V_{K}(t) \equiv\left(t^{1 / 2}+t^{-1 / 2}\right)^{r-1}\left[V_{k}(t)\right]^{r} \bmod \left(r,\left(t^{2 r}-t^{r+1}-t^{r-1}+1\right) /(t+1)\right) \text {. }
$$

Section 2 gives the proof of Theorem 1 and Theorem 2. In $\S 3$, we will present some examples and remarks.

I would like to express my appreciation to Professor Shin'ichi Suzuki for his helpful suggestions. And I would like to thank Professor Makoto Sakuma who gave me information about the criteria of periodic knots.

\section{Proof of Theorems 1 and 2}

Definition $1[1,4]$. Fix a nonzero complex number $v$ and a positive integer $n$. Then the Jones algebra $J_{n}$ is defined as a $C$-algebra with generators $1, e_{1}, e_{2}, \ldots, e_{n-1}$ and relations

$$
\begin{gathered}
e_{i}^{2}=-\left(v^{2}+v^{-2}\right) e_{i}, \\
e_{i} e_{i \pm 1} e_{i}=e_{i}, \\
e_{i} e_{j}=e_{j} e_{i} \text { if }|i-j|>1 .
\end{gathered}
$$

It is well known that $J_{n}$ is semisimple when $v$ is not a root of unity. Let $\rho_{n, i}(0 \leq i \leq[n / 2])$ be the irreducible representations of $J_{n}$, and $\chi_{n, i}(0 \leq$ $i \leq[n / 2])$ their characters.

Definition 2 [4]. Let $G_{n}$ be the free semigroup generated by $1, \epsilon_{i}, \sigma_{i}, \sigma_{i}^{-1}(1 \leq$ $i \leq n-1)$. For $\xi \in G_{n}$, we define an $n$-string tangle as in Figure 1 . We identify $\xi$ and this $n$-string tangle if there is no fear of confusion. By the analogy with braids, we define the closure of $\xi$, denoted by $\hat{\xi}$, naturally.

Let $\pi_{n}: G_{n} \rightarrow J_{n}$ be the semigroup homomorphism defined by $\pi_{n}\left(\epsilon_{i}\right)=e_{i}$, $\pi_{n}\left(\sigma_{i}\right)=v^{-1}+v e_{i}$ and $\pi_{n}\left(\sigma_{i}^{-1}\right)=v+v^{-1} e_{i}$. In [4], J. Murakami has shown the following. For $\xi \in G_{n}$,

$$
\langle\hat{\xi}\rangle=\sum_{i=0}^{[n / 2]} a_{n, i}(v) \chi_{n, i}\left(\pi_{n}(\xi)\right),
$$

where $\langle\hat{\xi}\rangle$ is the bracket polynomial [2] of $\hat{\xi}$ and

$$
a_{n, i}(v)=(-1)^{n+1}\left(v^{2 n+2-4 i}-v^{-2 n-2+4 i}\right) /\left(v^{4}-v^{-4}\right) .
$$

Now, we begin the proof of the theorems. From a simple observation, we can assume that there exists $\xi \in G_{n}$ such that $k=\hat{\xi}$ and $K=\hat{\xi}^{r}$. Then, $n$ is 


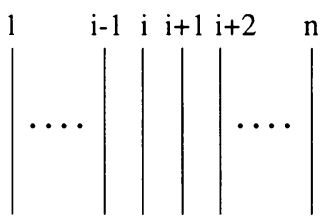

1

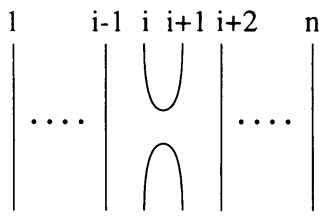

$\varepsilon_{\mathrm{i}}$

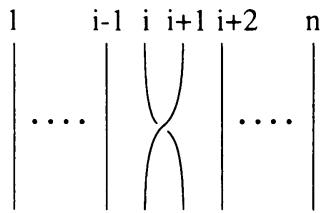

$\sigma_{\mathrm{i}}$

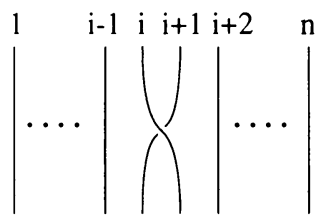

$\sigma_{i}^{-1}$

$$
\xi=\xi_{1} \xi_{2} \ldots \xi_{\mu-1} \xi_{\mu}
$$

where $\xi_{\mathrm{i}}$ is

$1, \varepsilon_{i}, \sigma_{i}$ or $\sigma_{i}^{-1}$

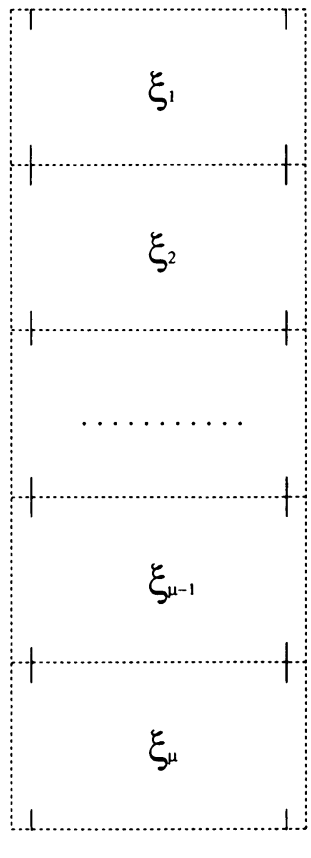

\section{FIGURE 1}

odd or even according as $\mathrm{k}(K, \operatorname{Fix}(f))$ is odd or even. Let $w(K)$ and $w(k)$ denote the writhes of $\hat{\xi}^{r}$ and $\hat{\xi}$ respectively. Then we have $w(K)=r w(k)$ and

$$
\begin{aligned}
& V_{K}\left(v^{4}\right)=\left(-v^{3}\right)^{w(K)}\left\langle\hat{\xi}^{r}\right\rangle=\sum_{i=0}^{[n / 2]} a_{n, i}(v)\left(-v^{3}\right)^{r w(k)} \chi_{n, i}\left(\pi_{n}\left(\xi^{r}\right)\right), \\
& V_{k}\left(v^{4}\right)=\left(-v^{3}\right)^{w(k)}\langle\hat{\xi}\rangle=\sum_{i=0}^{[n / 2]} a_{n, i}(v)\left(-v^{3}\right)^{w(k)} \chi_{n, i}\left(\pi_{n}(\xi)\right) .
\end{aligned}
$$

Let $f_{i}(v)=\left(-v^{3}\right)^{w(k)} \chi_{n, i}\left(\pi_{n}(\xi)\right) \in Z\left[v^{ \pm 1}\right]$.

Claim. $\left(-v^{3}\right)^{r w(k)} \chi_{n, i}\left(\pi_{n}\left(\xi^{r}\right)\right) \equiv f_{i}\left(v^{r}\right) \bmod r$. 
Proof. As $\rho_{n, i}\left(\pi_{n}(\xi)\right)$ is a matrix over $Z\left[v^{ \pm 1}\right]$, we have

$$
\begin{aligned}
\chi_{n, i}\left(\pi_{n}\left(\xi^{r}\right)\right) & =\operatorname{tr}\left(\rho_{n, i}\left(\pi_{n}\left(\xi^{r}\right)\right)\right) \\
& =\operatorname{tr}\left(\left(\rho_{n, i}\left(\pi_{n}(\xi)\right)\right)^{r}\right) \\
& \equiv\left(\operatorname{tr}\left(\rho_{n, i}\left(\pi_{n}(\xi)\right)\right)\right)^{r} \bmod r .
\end{aligned}
$$

That is, $\chi_{n, i}\left(\pi_{n}\left(\xi^{r}\right)\right) \equiv\left(\chi_{n, i}\left(\pi_{n}(\xi)\right)\right)^{r} \bmod r$. Therefore,

$$
\begin{aligned}
\left(-v^{3}\right)^{r w(k)} \chi_{n, i}\left(\pi_{n}\left(\xi^{r}\right)\right) & \equiv\left(\left(-v^{3}\right)^{w(k)} \chi_{n, i}\left(\pi_{n}(\xi)\right)\right)^{r} \\
& \equiv\left(f_{i}(v)\right)^{r} \\
& \equiv f_{i}\left(v^{r}\right) \bmod r .
\end{aligned}
$$

We now consider the two cases of theorems.

Case 1. $\operatorname{lk}(K, \operatorname{Fix}(f)) \equiv 1 \bmod 2$.

Note that $n$ is odd and $a_{n, i}(v) \in Z\left[v^{ \pm 4}\right]$ for every $i$. Write $f_{i}(v)=g_{i}(v)+$ $h_{i}(v)$ where $g_{i}(v)$ and $h_{i}(v)$ are elements of $Z\left[v^{ \pm 4}\right]$ and $Z\left[v^{ \pm 1}\right]-Z\left[v^{ \pm 4}\right]$ respectively. By the claim, we have

$$
V_{K}\left(v^{4}\right) \equiv \sum_{i=0}^{[n / 2]} a_{n, i}(v) g_{i}\left(v^{r}\right)+\sum_{i=0}^{[n / 2]} a_{n, i}(v) h_{i}\left(v^{r}\right) \bmod r
$$

and

$$
V_{k}\left(v^{4}\right)=\sum_{i=0}^{[n / 2]} a_{n, i}(v) g_{i}(v)+\sum_{i=0}^{[n / 2]} a_{n, i}(v) h_{i}(v)
$$

As $K$ and $k$ are knots, $V_{K}\left(v^{4}\right)$ and $V_{k}\left(v^{4}\right)$ are in $Z\left[v^{ \pm 4}\right]$. That is,

$$
\sum_{i=0}^{[n / 2]} a_{n, i}(v) h_{i}\left(v^{r}\right) \equiv \sum_{i=0}^{[n / 2]} a_{n, i}(v) h_{i}(v) \equiv 0 \bmod r .
$$

From $a_{n, i}(v)=a_{n, i}\left(v^{-1}\right)$, we have

$$
V_{K}\left(v^{4}\right)-V_{K}\left(v^{-4}\right) \equiv \sum_{i=0}^{[n / 2]} a_{n, i}(v)\left(g_{i}\left(v^{r}\right)-g_{i}\left(v^{-r}\right)\right) \bmod r .
$$

Since each $g_{i}\left(v^{r}\right)$ is an element of $Z\left[v^{ \pm 4 r}\right], v^{4 r}-v^{-4 r}$ divides $g_{i}\left(v^{r}\right)-g_{i}\left(v^{-r}\right)$. By replacing $t$ for $v^{4}$, we obtain Theorem $1(\mathrm{i})$. Furthermore

$$
V_{K}\left(v^{4}\right)-\left[V_{k}\left(v^{4}\right)\right]^{r} \equiv \sum_{i=0}^{[n / 2]}\left(a_{n, i}(v)-\left(a_{n, i}(v)\right)^{r}\right) g_{i}\left(v^{r}\right) \bmod r
$$

It is easy to show $a_{n, i}(v)-\left(a_{n, i}(v)\right)^{r} \equiv 0 \bmod \left(r, v^{8 r}-v^{4 r+4}-v^{4 r-4}+1\right)$. A substitution $t$ for $v^{4}$ gives Theorem 2(i).

Case 2. $\operatorname{lk}(K, \operatorname{Fix}(f)) \equiv 0 \bmod 2$. 
Note that $n$ is even and that $a_{n, i}(v) \notin Z\left[v^{ \pm 4}\right]$ for every $i$. However $\left(v^{2}+v^{-2}\right) a_{n, i}(v)$ is in $Z\left[v^{ \pm 4}\right]$ for every $i$. By the argument similar to that of Case 1, we obtain

$$
\begin{aligned}
& \left(v^{2}+v^{-2}\right) V_{K}\left(v^{4}\right) \equiv \sum_{i=0}^{[n / 2]}\left(v^{2}+v^{-2}\right) a_{n, i}(v) y_{i}\left(v^{r}\right) \bmod r \\
& \left(v^{2}+v^{-2}\right) V_{k}\left(v^{4}\right)=\sum_{i=0}^{[n / 2]}\left(v^{2}+v^{-2}\right) a_{n, i}(v) y_{i}(v)
\end{aligned}
$$

where each $y_{i}(v)$ is in $Z\left[v^{ \pm 2}\right]-Z\left[v^{ \pm 4}\right]$. Therefore we have

$$
\begin{aligned}
\left(v^{2}+\right. & \left.v^{-2}\right)\left(V_{K}\left(v^{4}\right)-V_{K}\left(v^{-4}\right)\right) \\
& \equiv \sum_{i=0}^{[n / 2]}\left(v^{2}+v^{-2}\right) a_{n, i}(v)\left(y_{i}\left(v^{r}\right)-y_{i}\left(v^{-r}\right)\right) \bmod r, \\
\left(v^{2}+v^{-2}\right)\left(V_{K}\left(v^{4}\right)+V_{K}\left(v^{-4}\right)\right) & \\
& \equiv \sum_{i=0}^{[n / 2]}\left(v^{2}+v^{-2}\right) a_{n, i}(v)\left(y_{i}\left(v^{r}\right)+y_{i}\left(v^{-r}\right)\right) \bmod r .
\end{aligned}
$$

Since each $y_{i}\left(v^{r}\right)$ is an element of $Z\left[v^{ \pm 2 r}\right]-Z\left[v^{ \pm 4 r}\right], v^{2 r}-v^{-2 r}$ and $v^{2 r}+v^{-2 r}$ divide $y_{i}\left(v^{r}\right)-y_{i}\left(v^{-r}\right)$ and $y_{i}\left(v^{r}\right)+y_{i}\left(v^{-r}\right)$ respectively. By replacing $t$ for $v^{4}$, we obtain Theorem 1(ii). Furthermore,

$$
\begin{aligned}
\left(v^{2}\right. & \left.+v^{-2}\right) V_{K}\left(v^{4}\right)-\left[\left(v^{2}+v^{-2}\right) V_{k}\left(v^{4}\right)\right]^{r} \\
& \equiv \sum_{i=0}^{[n / 2]}\left(\left(v^{2}+v^{-2}\right) a_{n, i}(v)-\left(\left(v^{2}+v^{-2}\right) a_{n, i}(v)\right)^{r}\right) y_{i}\left(v^{r}\right) \bmod r .
\end{aligned}
$$

It is also easy to show that

$$
\left(v^{2}+v^{-2}\right) a_{n, i}(v)-\left(\left(v^{2}+v^{-2}\right) a_{n, i}(v)\right)^{r} \equiv 0 \bmod \left(r, v^{8 r}-v^{4 r+4}-v^{4 r-4}+1\right) .
$$

A substitution $t$ for $v^{4}$ gives Theorem 2(ii).

\section{EXAMPLES}

We begin with the following proposition.

Proposition. For any knot $K$, either

(i) $V_{K}(t)-V_{K}\left(t^{-1}\right) \equiv 0 \bmod \left(t^{6}-1\right)$ or

(ii) $V_{K}(t)+V_{K}\left(t^{-1}\right) \equiv 0 \bmod \left(t^{2}-t+1\right)$.

Proof. Let $V_{K}(t)=\left(t^{2}-t+1\right) Q(t)+a t+b, a, b \in Z$. As $V_{K}\left(e^{\pi i / 3}\right)$ is a power of $i \sqrt{3}$ [3], we have $a=0$ or $a+2 b=0$. If $a+2 b=0$, a simple calculation shows (ii). If $a=0$, it is clear that $t^{2}-t+1$ divides $V_{K}(t)-V_{K}\left(t^{-1}\right)$. Furthermore $t^{3}-1$ and $t+1$ also divide $V_{K}(t)-V_{K}\left(t^{-1}\right)$ [1]. This shows (i). 
By the proposition, it seems that Theorem 1 does not work in the case $r=3$. But we know Murasugi's result [5]:

$$
\Delta_{K}(t) \doteq \Delta_{k}(t)^{r}\left(1+t+\cdots+t^{\lambda-1}\right)^{r-1} \bmod r,
$$

where $\Delta_{K}(t)$ and $\Delta_{k}(t)$ are the Alexander polynomials of $K$ and $k$, respectively, and $\lambda=\operatorname{lk}(K, \operatorname{Fix}(f))$. So we can evaluate $\operatorname{lk}(K, \operatorname{Fix}(f))$ from $\Delta_{K}$ and there is a possibility that Theorem 1 works in the case $r=3$. But the author does not have such an example. In the cases $r \geq 5$, Theorem 1 works well. Here we give two examples.

Example 1. Consider $K=10_{24}$. Traczyk's criterion does not work for $r=5$ because $V_{K}(t)-V_{K}\left(t^{-1}\right) \equiv 0 \bmod \left(5, t^{5}-1\right)$. From $\Delta_{K}(t) \doteq(1+t)^{4} \bmod 5$, if $K$ has period $5, \mathrm{lk}(K, \operatorname{Fix}(f))$ must be 2 . But we have $V_{K}(t)+V_{K}\left(t^{-1}\right) \not \equiv$ $0 \bmod \left(5,\left(t^{5}+1\right) /(t+1)\right)$. By Theorem $1, K$ cannot have period 5.

Example 2. Consider $K=10_{55}$. Traczyk's criterion also does not work for $r=5$. From $\Delta_{K}(t) \doteq 1 \bmod 5$, if $K$ has period $5,1 \mathrm{k}(K, \operatorname{Fix}(f))$ must be 1 . But we have $V_{K}(t)-V_{K}\left(t^{-1}\right) \not \equiv 0 \bmod \left(5, t^{10}-1\right)$. By Theorem $1, K$ can not have period 5.

Finally we remark that Theorem 2 holds for the Jones polynomial of periodic links.

\section{REFERENCES}

1. V. F. R. Jones, Hecke algebra representations of braid groups and link polynomials, Ann. of Math. 126 (1987), 335-388.

2. L. H. Kauffman, State models and the Jones polynomial, Topology 26 (1987), 395-407.

3. W. B. R. Lickorish and K. C. Millett, Some evaluations of link polynomials, Comment. Math. Helv. 61 (1986), 349-359.

4. J. Murakami, The parallel version of polynomial invariants of links, Osaka J. Math. 26 (1989), 1-55.

5. K. Murasugi, On periodic knots, Comment. Math. Helv. 46 (1971), 162-174.

6. ___ Jones polynomials of periodic links, Pacific J. Math. 131 (1988), 319-329.

7. P. Traczyk, $10_{101}$ has no period 7: a criterion for periodicity of links, Proc. Amer. Math. Soc. 108 (1990), 845-846.

Department of Mathematics, School of Science and Engineering, Waseda University, OHKubo, ShinJuku Tokyo, 169, JAPAN 